\title{
Risk factors and diagnosis of cerebral venous thrombosis: Data from a cohort of $\mathbf{4 5}$ Romanian patients
}

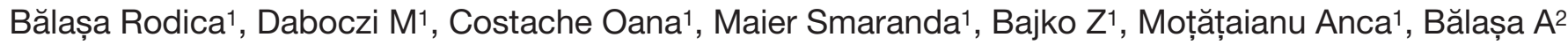 \\ ${ }^{1}$ First Neurological Clinic, Regional MS Centre, University Emergency County Hospital, Târgu Mureș, University of Medicine and Pharmacy Târgu Mureș, \\ România \\ 2 Neurosurgery Department, University Emergency County Hospital Târgu Mureș, România
}

Cerebral venous thrombosis (CVT) represents $1 \%$ of the total stroke pathology but is a real challenge both regarding the diagnosis and the treatment.

Objective: Evaluate different etiological, demographical, clinical, imaging and therapeutic aspects of CVT.

Material: Prospective study during 4 years. From the total 3658 patients hospitalized with acute stroke, 45 (1.23\%) had CVT. For each patient, were recorded: demographic data, symptom of onset, type of onset, daily habits, medical history, neurological examination, brain imaging (CT and MRI with venography). Statistical analysis: data are presented as mean and SD and Student $t$ test was applied.

Results: Mean age was 44.07 $\pm 23,12$ years; female: male ratio 2.21:1. The most frequent type of onset was acute (77.78\%). Headache was found in $80 \%$ of cases as initial symptom, followed by neurological focal deficits. As risk factors, thrombophilia was found most often (59.5\%), followed by local infections. No risk factors were found in $17.8 \%$ of cases. The brain imaging was positive in 29 patients. In 16 cases, the imaging workout was negative and the diagnosis consisted of clinical criteria, risk factors, response to heparin treatment.

Conclusions: CVT is a rare pathology that affects mainly young women and that needs a complex diagnostic evaluation. The patient prototype diagnosed with CVT in our region: female of 44 years old, with an intense acute headache, with MRI showing direct signs of transverse sinus thrombosis, with a thrombophilic state and good response to anticoagulants. Brain MRI is the imaging investigation required but clinical aspects play a decisive role.

Keywords: cerebral venous thrombosis, venous infarction, MR venography, CT venography.

Received: 16 October 2014 / Accepted: 25 October 2014

\section{Introduction}

Cerebral venous thrombosis (CVT) represents approximately $1 \%$ of all types of stroke but it is more difficult both to diagnose, to perform the etiological workout and also to treat. Due to the new and modern means of investigation (mostly the development of neuroimaging) the term of CVT hides a wider clinical picture than initially considered. Clinical aspects of CVT are represented by: headache, papilledema, visual loss, focal or generalized seizures, focal neurologic deficits, confusion, altered consciousness and coma $[1,2]$.

Symptoms associated with CVT are related to the area/ sinus of thrombosis, requiring a good knowledge of the anatomy of venous system as an essential step in evaluation of these patients. An early therapeutic measure is easier nowadays due to the progress of neuroimaging investigations $[3,4]$.

The incidence of CVT is difficult to determine, but generally it is believed to represent an uncommon cause of stroke, with the reported ratio of venous to arterial strokes being 1:62.5. Nowadays the incidence is estimated to be

Correspondence to: Rodica Bălașa

E-mail: iipascu@redatronic.ro around $0.22 / 100,000$ annually in adults, 0.67 cases per 100000 in children under 18 years and 11.6/100,000 in obstetric patients. It can pose diagnostic and therapeutic challenges and without a high level of suspicion, can be easily missed or mistaken for other, more frequent neurological conditions. The mortality from CVT, classically ranging from 30 to $50 \%$, dropped nowadays to less than $10 \%[2-15]$.

The pathogenesis of CVT is still in debate but 2 mechanisms have demonstrated a constant implication in disease onset: a) elevated intracranial pressure (decrease in cerebrospinal fluid absorption); b) cerebral parenchymal lesions (secondary to thrombosis of dural sinuses and/or cerebral veins) $[6,12,14-16]$.

Regarding the etiology of CVT, many risk factors are found to play a major or a secondary role. The major risk factors are found in more than $85 \%$ of cases, being represented by: oral contraceptives, pregnancy and puerperium, malignancy, protrombotic genetic (G20210 A protrombin gene mutation, factor $V$ Leiden mutation, MTHFR gene mutation etc) or acquired (antithrombindeficiency, protein $\mathrm{C}$ or $\mathrm{S}$ deficiency, hiperhomocysteinemia, etc) conditions. The implications of genetic and some behaviour factors explain the different aetiologies found in different ethnic 
and population groups. These differenced determined us to look carefully in our CVT patient group [1,2,9,10,17-36].

The most sensitive imaging technique to confirm the diagnosis of CVT is magnetic resonance imaging (MRI) with acquisition of venography sequences. This technique is more sensitive than brain computer tomography (CT) [37-48].

The treatment of CVT is mainly based on the use of anticoagulants, treatment of risk factors and decrease of an elevated intracranial pressure. The use of long term oral anticoagulants is still in debate, but it is generally agreed that after 6 months of anticoagulation their use must be restricted only to patients that have a proved thrombophilic state [49-54].

\section{Material and Methods}

We performed a prospective study that took place in the First Neurological Clinic from Târgu-Mureș during a period of 4 years: July 2010 - July 2014. From the total of 3658 patients that were hospitalized during this period with acute stroke, 45 (1.23\%) had a diagnosis of CVT.

Our objective was to evaluate different etiological, demographical, clinical and imaging aspects of the CVT as well as response to treatments within this condition.

Inclusion criteria: positive diagnosis of CVT, according to the four syndromes that determine the clinical presentations proposed by Bousser and Ferro in 2007 [1]: a) focal syndrome (focal signs together with headache or/and seizures or/and various degrees of altered consciousness); b) intracranial hypertention syndrome (headache, nausea, vomiting, papillary oedema); c) subacute diffuse encephalopathy (various degrees of altered consciousness); d) cavernous sinus syndrome (ophtalmoplegia, proptosis, kemosis, dyplopia). All these clinical criteria were interpreted together with the symptomatology and different cerebral imaging techniques such as: MRI with venography, CT with venous acquisition and in some patients classical venography.

Exclusion criteria: patients younger than 18 years old, other pathologies sometimes with similar symptomatology but with positive cerebral imaging for: arterial stroke, primary or metastatic tumours, different demyelinating diseases including multiple sclerosis, arterio-venous malformations, and different cerebral infections. We also excluded patients in whom the differential diagnosis workout was not finished.

For each patient, demographic date were recorded together with: onset of symptoms, type of onset, daily habits (smoking, alcohol intake), medical history (including oral contraceptive consumption, cerebral trauma, previous surgery, infections mainly in the facial region, pregnancy and postpartum period, presence of autoimmune diseases, presence of chronic infections like HIV, hepatitis type B or syphilis), neurological examination, brain imaging which consisted in CT with venography in all 45 patients, while 31 cases $(68.88 \%)$ had also a brain MRI with gadolinium
(Gd) contrast administration and image acquisition of both parenchymal and cerebral venous sinuses and veins. In a case where a strong clinical suspicion of CVT was raised and both CT and MRI were negative, a classical venography was performed that finally confirmed the diagnosis. In both CT and MRI imaging methods, the presence of direct signs for CVT (presence of dense clot, cord sign, empty delta sign, absence of normal flow void in affected veins on MRI technique), as well as the presence of indirect signs (venous infarction with or without secondary bleeding, cortical oedema, cortical/cerebral haemorrhage) was marked for each patient. The CT was performed in emergency conditions while the MRI examination was performed the next day in some cases.

All patients had also an ocular fundoscopy performed either by the neurologist or by the ophthalmologist. In all patients a wide paraclinical investigation was performed in order to identify the risk factors for the CVT: diagnosis of autoimmune connective diseases (Sjögren syndrome, systemic lupus erythematosus, anti-phospholipid antibodies, sarcoidosis, cerebral vasculitis), HIV1 or 2 serology, Treponema pallidum haemagglutination assay, other tests for thrombophilia (serum level of protein C, S, antithrombin III, G20210 A protrombin gene mutation, factor $\mathrm{V}$ Leiden mutation, MTHFR gene mutation etc).

Statistical analysis: Parametric data are presented as mean and SD and Student t-test was used for statistical analysis. Differences between variables were evaluated for significance using t-tests or Wilcoxon's rank-sum tests. These were considered significant if the $\mathrm{p}$ value was $<0.05$.

We focused mainly on the clinical aspects of the onset in CVTs, correlated with the neuroimaging that confirmed the diagnostic and with the risk factors found.

The study respected the Declaration of Helsinki. All subjects or their first degree relatives signed an informed consent that was approved by the local ethics committee.

\section{Results}

From the total of 3658 cases of acute stroke that were admitted in our department in the study period, $45(1,23 \%)$ cases were diagnosed with CVT.

The mean age of patients with CVT was $44.07 \pm 23.12$ years (minimum 20 years, maximum 81years old). Almost half of the patients $(46.67 \%)$ were in the $30-50$ years old group, $33.33 \%$ were older than 50 and the rest of $20 \%$ of cases were younger than 30 years old.

The female: male ratio was 2.21:1. Regarding the sex distribution in the three age groups, the most important significant difference was found in the youngest group of patients $(<30$ years of age) where the female to male ratio was 5:1, while in the oldest group the number of females was equal to those of men (6 each).

The most frequent type of onset was, as we expected, acute (less than 48 hours, 35 cases $-77.78 \%$ ), followed by subacute onset (2-30 days with a mean time of onset at approximately 1 week since the first signs appeared) in 7 cases 
(15.56\%), while 3 patients (6.67\%) had a chronic type of onset (more than 30 days).

Among the most important three symptoms presented by the patients, headache was found in $80 \%$ of cases, followed by neurological focal deficits in $48.9 \%$ of patients and nausea, which was described by $28.9 \%$ of patients. Interestingly, generalized seizures and papillary oedema that were previously presented as main CVT symptoms were found in our cohort only in $17.8 \%$ and $15.5 \%$ respectively of cases. Figure 1 presents the frequency of signs and symptoms found at CVT onset.

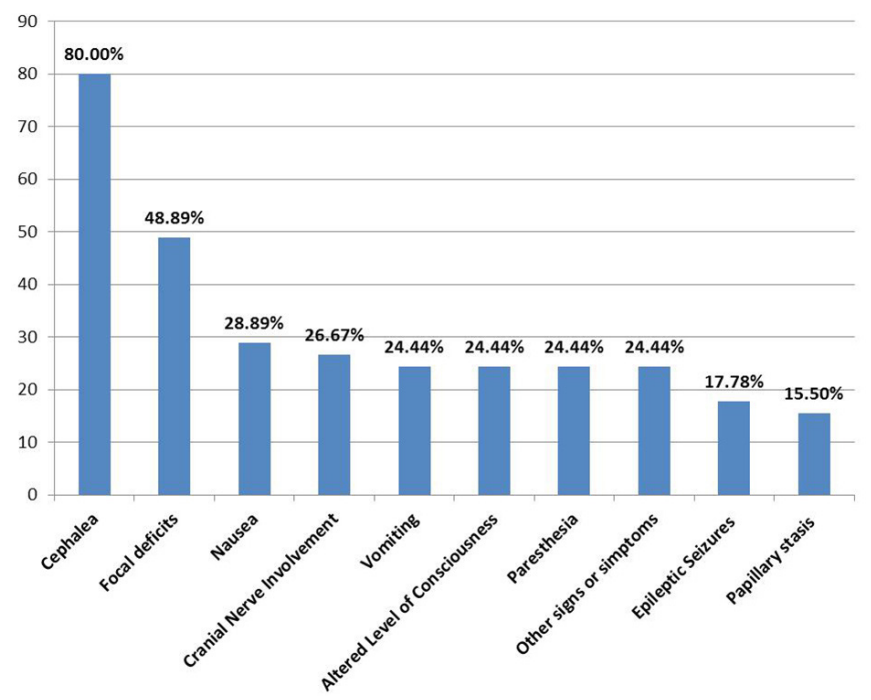

Fig. 1. Clinical manifestations in the CVT cohort.

The risk factors were searched and divided in a systematic manner: systemic risk factors (thrombophilia inherited or acquired, smoking, previous surgery or trauma, pregnancy and postpartum period, etc.) and local risk factors such as local infections. Table I shows the risk factors found in our cohort of CVTs (whether a systemic or local cause was discovered). Thrombophilia was found most often in 22 cases $(59.5 \%)$, followed by local infections like otomastoiditis in 12 patients (32.43\%) and by smoking. In a significant percentage of cases $(17.8 \%)$ we could not identify any risk factor.

Regarding the brain imaging, this method was useful in 29 patients contributing both to diagnose CVT and to clarify the topography of the affected vein/veins or venous sinuses (tab. II). In 16 cases, the imaging workout was negative and the diagnosis consisted of clinical criteria (signs of intracranial hypertension in $18.75 \%$ of patients), presence of risk factors (thrombophilia in $43.75 \%$ of cases, local infection in $18.75 \%$ of cases) and response to heparin treatment. The main imagistic method used in our patients to confirm the diagnostic and also to rule out other diseases was CT that was performed in all 45 patients and was suggestive for CVT in 13 cases (35.2\%) (fig. 2). MRI was done in 31 patients and was suggestive for CVT in 19 cases (61.3\%) (fig. 3, fig. 4). In one case the classical angiography/venography confirmed the previous suspicion of CVT.
Fig. 5 graphically presents the case distribution according to the direct/indirect imaging signs fond on CT or/and MRI. The most frequent sinus involvement was found in transversal sinus (44.4\%), followed by cortical veins in $24.4 \%$ and then thrombosis of the sagittal sinus (20\%). Other imaging signs of CVT were found with the following localisation: cavernous sinus (15.56\%), sigmoid sinus $(11.1 \%)$ and straight sinus in one patient (2.2\%) (fig. 6).

Regarding the treatment, all patients were put on anticoagulant therapy that consisted in the acute phase of heparin or of derivates of heparin with low molecular weight

Table I. Risk factors in the CVT cohort.

\begin{tabular}{ccc}
\hline Risk factors & Patients number & Percent \\
\hline Thrombophilia & 22 & $59.46 \%$ \\
Infections & 12 & $32.43 \%$ \\
Smoking & 11 & $29.73 \%$ \\
Pregnancy and puerperium & 6 & $27.27 \%$ * \\
Previous surgery & 6 & $16.22 \%$ \\
Oral contraceptive intake & 3 & $13.64 \%$ * \\
Trauma & 2 & $5.41 \%$ \\
Without evident risk factors & 8 & $17.78 \%$ \\
\hline
\end{tabular}

* percentage calculated only in female patients under 50 years of age

Table II. Brain imaging in the CVT cohort

\begin{tabular}{cccc}
\hline Imaging & $\begin{array}{c}\text { Number } \\
\text { of } \\
\text { patients }\end{array}$ & $\begin{array}{c}\text { Number of patients } \\
\text { with positive } \\
\text { imaging for CVT }\end{array}$ & $\begin{array}{c}\text { Porcentage of } \\
\text { patients with } \\
\text { positive CVT exam. }\end{array}$ \\
\hline $\begin{array}{c}\text { Cerebral } \\
\text { Angiography }\end{array}$ & 1 & 1 & $100.00 \%$ \\
MRI & 31 & 19 & $61.29 \%$ \\
CT & 37 & 13 & $35.14 \%$ \\
\hline
\end{tabular}

(enoxaparin, nadroparin). In 55.56\% of cases Mannitol was used in order to reduce intracranial tension and with the same purpose we used various types of corticoid treatment (Dexamethasone or methylprednisolone) in 37.78\% of patients. Antiepileptic drugs (levtiracetam, carbamazepine, valproic acide) were used only in patients that had seizures $(33.33 \%)$. We also administered symptomatic therapy like analgesics in $53.3 \%$ of patients. At discharge, the patients were put on oral anti-K vitamin therapy for at least 6 months (patients that had an inherited thrombophilia remained on anticoagulant therapy for longer periods).

We had no fatal events, 2 patients remained tetraparetic and the rest of 43 had a regressive symptomatology or even asymptomatic at discharge.

\section{Discussions}

CVT is an uncommon and frequently unrecognized type of stroke that affects approximately 5 people per million annually and accounts for $0.5 \%$ to $1 \%$ of all strokes [1-3]. The results are similar to our findings (approximately 1\%). CVT is more commonly seen in young female individuals. According to the largest cohort study, 487 (78\%) of 624 cases of CVT occurred in patients $<50$ years of age [1]. The mean age of our series ( 44 years) were slightly different from the literature data : 37 years [53] and 43 years [13]. 

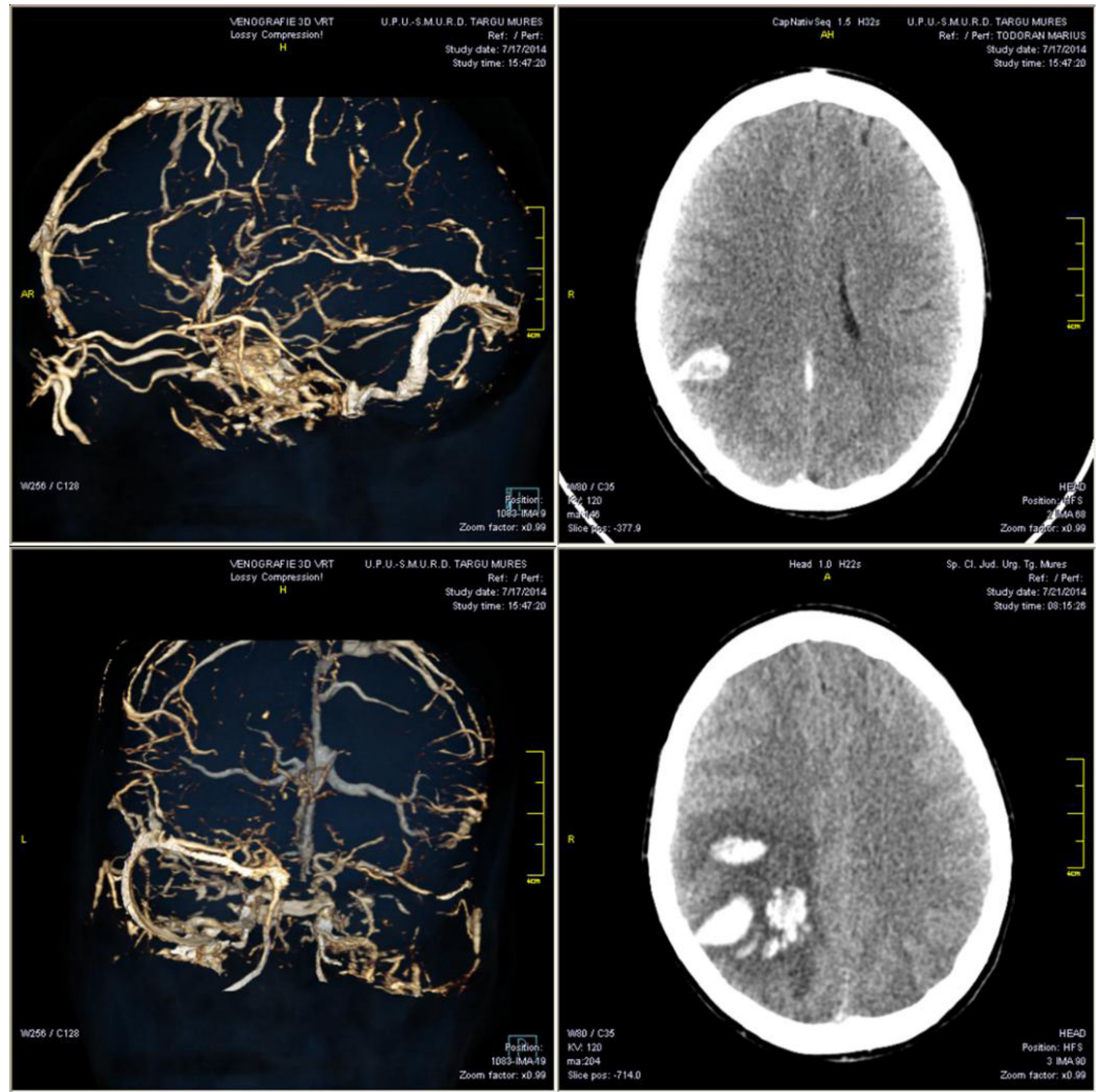

Fig. 2. Brain CT scan and CT venography in a 33 years old female patient with CVT. Lack of visualization (direct sign of CVT) of the posterior part superior sagittalis sinus, inferior sagittalis sinus, sinus rectus, right sided sinus transversus and sigmoideus. Hemorrhagic venous infarction (indirect sign of CVT) in the posterior part of the right parietal region.

We had a significant difference in the CVT incidence in patients younger than 50 years old. In the literature the proportion of CVT patients that present this age criterion represents $78 \%[1,54]$ while in our study the percentage was slightly lower, $67 \%$. This might be due to a higher access to MRI in this active group of women from western countries compared with our group.

CVT is believed to be more common in women than in men. In a series of 110 cases, Ameri and Bousser [16] found a female-to-male ratio of 2.29:1. In almost total accordance with the above mentioned publication we found a female-to-male ratio of 2.21:1.
The onset of the cerebral venous thrombosis has a wide distribution. We found that in $77.78 \%$ of the cases included, the onset was acute while data from the literature show for this kind of onset very different results: $28 \%[2,16]-$ $37.2 \%$ [54]. The subacute onset was found in our cohort in $15.56 \%$ of cases while in other studies this type of onset was the most frequently found, being $42 \%$ in Ameri and Bousser [16] study. The chronic type of onset was found in $6.69 \%$ in our cases compared with $7.2 \%$ in the study conducted by Ferro et al [54]. A difference in our study series was observed, mostly related to the fact that we had a higher number of patients with acute onset. This fact might 


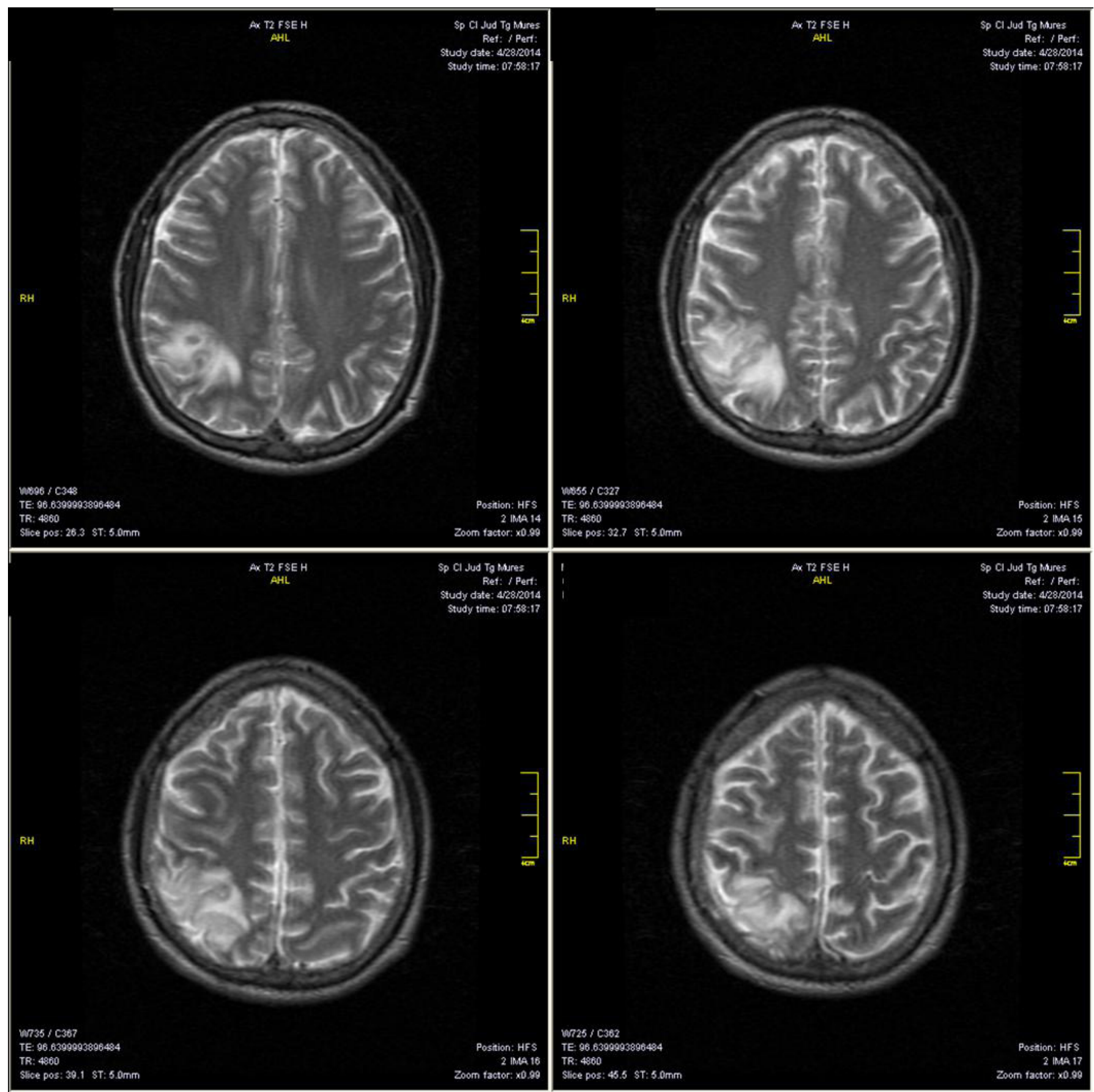

Fig. 3. Brain MRI (T2 sequences) in a 30 years old male patient with sinus sagittalis superior thrombosis with right parietal infarction (indirect signs).

be explained by the high addressability of the patients to the emergency room when a sudden atrocious headache interferes.

As shown in fig. 1, the majority $(80 \%)$ of our patients accused headache as the first symptom, almost a half presented focal neurological deficits, while only $17.8 \%$ had the classical presentation with seizures. Ferro et al [54] and other studies $[7,24,55]$ found that the most common presenting symptom was headache (80-90\%) that could be the only symptom in up to $5 \%$ of cases. In many studies, seizures (focal or generalized) and paresis were seen in up to $40 \%$ of patients $[1,2,3,8,10,16]$. Table III presents data from our study compared with those found by Ferro et al in 2004 [54].
Table III. Clinical aspects of CVT onset: comparison between study data and the literature.

\begin{tabular}{lll}
\hline $\begin{array}{l}\text { Sign and symptoms from } \\
\text { the CVT onset }\end{array}$ & Personal Data & Data from the literature \\
\hline Headache & $80 \%$ & $70 \%[14]-88.8 \%[40]$ \\
Focal neurological deficit & $48.9 \%$ & $37.9[14]-40[40]$ \\
Papilary oedema & $38.9 \%$ & $28.3[40]$ \\
Cranial nerve involvement & $26.67 \%$ & $15 \%[40]$ \\
Altered consciousness & $24.4 \%$ & $22[40]-25.5 \%[15]-$ \\
Paresthesia & $24.4 \%$ & $47.5 \%[13]$ \\
Epileptic seizures & $17.78 \%$ & $5.4 \%[40]$ \\
& & $14.9[15]-39.3 \%[40]-$ \\
\hline
\end{tabular}




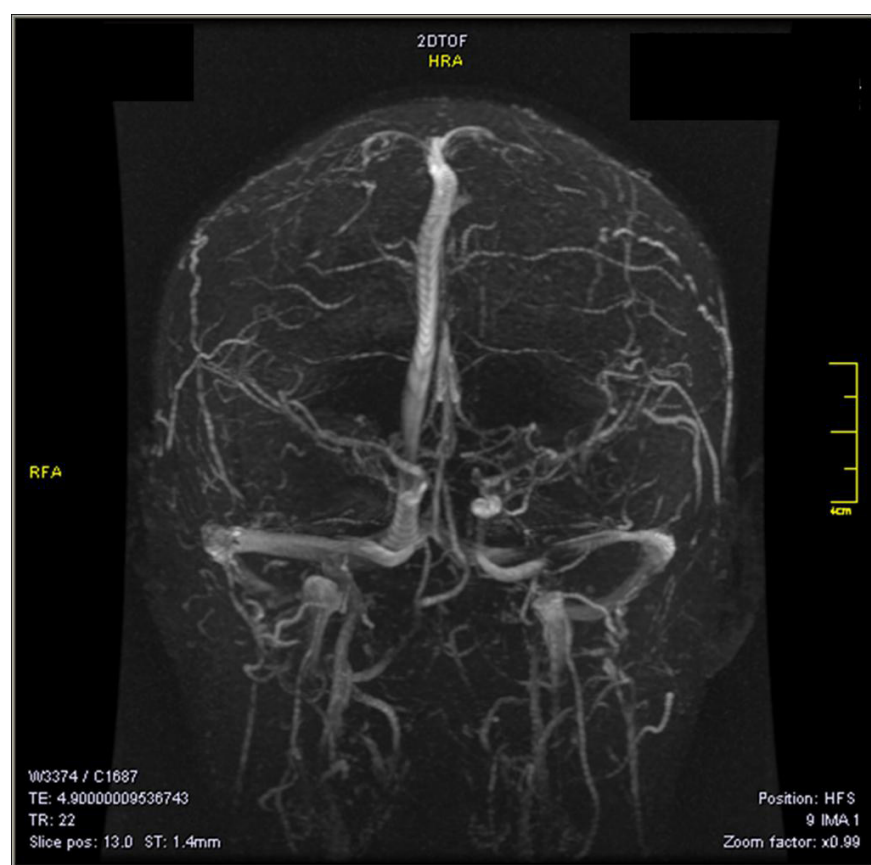

Fig. 4. MRI venography: partial thrombosis of the left transversal sinus (direct sign) in a 34 years old male with sinusitis and otomatoiditis.

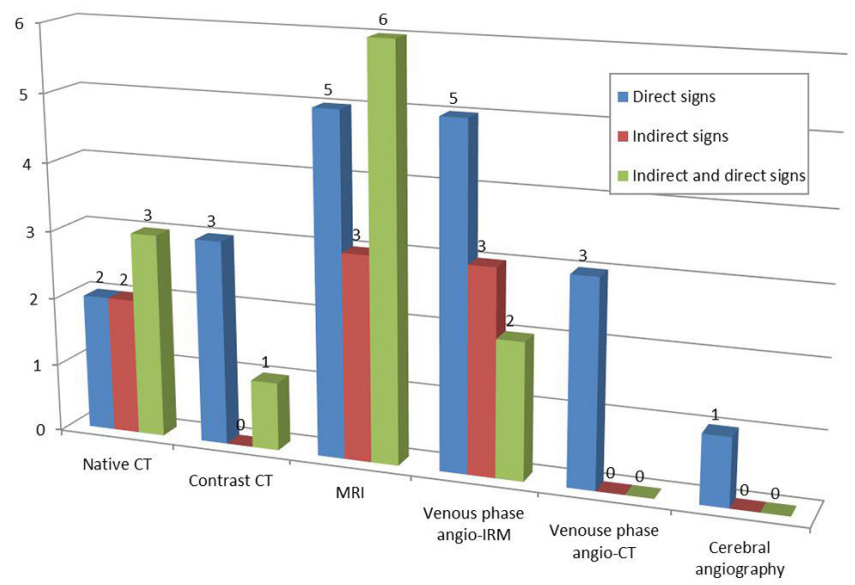

Fig. 5 Direct and indirect signs of CVT seen on brain imaging.

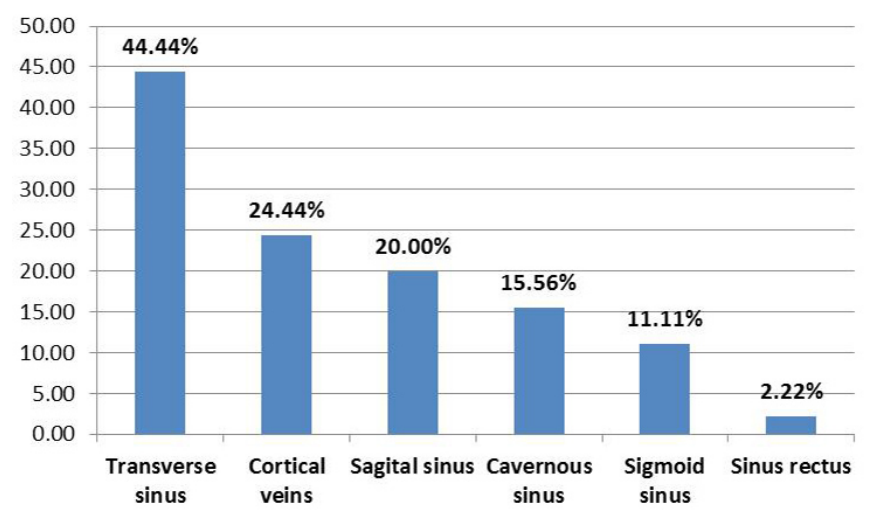

Fig. 6 Sinuses and veins involvement in CVT patients.
Table IV. Sinus and venous involvement in CVT: comparision between study data and the literature.

\begin{tabular}{ccc} 
Localization & $\begin{array}{c}\text { Personal } \\
\text { data }\end{array}$ & Data from the literature \\
\hline Transvers Sinus & $44.44 \%$ & $47.00 \%[58]-61.70 \%[15]-66.40 \%[14]$ \\
Cortical Veins & $24.44 \%$ & $6.00 \%[58]-17.10 \%[40]$ \\
\hline $\begin{array}{c}\text { Superior sagital } \\
\text { sinus }\end{array}$ & $20.00 \%$ & $44.70 \%[15]-62.00 \%[40]-71.00 \%[58]$ \\
Cavernous sinus & $15.56 \%$ & $1.3 \%[40]-31.00 \%[58]$ \\
\hline Sigmoid sinus & $11.11 \%$ & $31.00 \%[58]-74.50 \%[15]$ \\
\hline Rectus sinus & $2.22 \%$ & $10.00 \%[58]-18.00 \%[40]$ \\
\hline
\end{tabular}

Regarding the major risk factor for CVT inherited and/ or acquired hypercoagulability disorder was predominant in our study $(59.46 \%)$, as well as in the literature $[2,56]$. Venous thrombosis occurs if there is an exaggerated activity of haemostasis and/or a reduced activity of the antithrombotic mechanisms. Still CVT has in most cases a heterogenous ethiology and pathogenesis. Most frequently, a hereditary thrombophilias (factor V Liden, hereditary hiperhomocisteinemia, deficiency of Protein C, Protein S, antithrombin III, increased factor VIII and dysfibrinogenemia) is associated to one or more acquired causes (dehydration, surgery, trauma, pregnancy/postpartum period, hematological disorders, vicinity infections, autoimmune diseases like antiphospholipid antibodies, renal, cardiac, liver diseases, increased age, malignances, smoking), corticosteroid treatment, lumbar puncture. In most cases, one or more acquired causes are the real cause for CVT. In favor of this statement, stand the numerous causes of acquired Protein $C$ and $S$ and antithrombin deficiency in liver diseases, elevated factor VIII is found in obese and aged patients, hiperhomocisteinemia has a multitude of causes (malnutrition, Vitamine B12 and folic acid deficiency), etc. Local infections were almost double in frequency in our study compared with other data published. The explanation might be in the high percentage of patients that neglect the ear or sinus infections. Also a difference but in a different direction is the oral contraceptive use as a risk factor in CVT. In our study the number of patients found with this risk factor is reduced to a third than in other cohorts. The explanation might be that this medication is not used on a wide scale by our female population as a contraceptive measure. Studies made in countries from the Middle East had the same percentage of patients using oral contraceptives as we found in our study $[5,14,16,17$ $22,56,57]$.

The thrombus was most often localized in the transverse sinus in our study, like in majority of other published data. In the literature, the second sinus affected in CVT is the sagittal sinus with a range frequency of $44 \%-71 \%$, while in our study was found to be affected in $20 \%$ of cases. The differences among these studies are presented in table no IV. A very important issue is the discussion regarding the best examination for the positive diagnosis of CVT. In our data, together with data that we found in literature, brain CT imaging is positive only in approximately $1 / 3$ of cases. 
The gold standard method used was cerebral MRI that was positive in more than $60 \%$ of cases. In some studies that beneficiated of a very modern MRI machines, the percentage grew up to $70 \%$. We can also notice that one third of patients can have CVT in the presence of normal brain CT and MRI, the importance of clinical aspects in this pathology is major $[40,54]$.

Despite the high rate of mortality in patients with CVT, the outcome of our patients was good.

\section{Conclusions}

CVT is a rare pathology that affects mainly young women and needs a more complex diagnostic evaluation than the rest of stroke pathology. The CVT etiology is multifactorial and identification of all risk factors is essential for the long term prevention and treatment. The patient prototype diagnosed with CVT in our clinic has the following characteristics: a female of 44 years old, presenting an intense headache with acute onset, with an MRI showing direct signs of transverse sinus thrombosis, with a thrombophilic state and with good response to anticoagulants. Brain MRI is the main imagistic investigation required in a case of CVT but the clinical aspects play a decisive role.

\section{Conflict of interest}

Authors have nothing to declare.

\section{References}

1. Bousser MG, Ferro JM. Cerebral venous thrombosis: an update. Lancet Neurol. 2007;6: 162-170.

2. Karam C. Cerebral venous and sinus thrombosis in adults: a practical approach. Internet J Neurol 2007;10: 1-10

3. Guenther G, Arauz A. Review cerebral venous thrombosis: a diagnostic and treatment update. Neurologia 2011;26:488-498.

4. Saposnik G, Barinagarrementeria F, Brown RD Jr, et al. Diagnosis and management of cerebral venous thrombosis: a statement for healthcare professionals from the American Heart Association/ American Stroke Association. Stroke 2011; 42:1158-1192.

5. Jia LY, Hua Y, Ji XM et al. Correlation analysis of internal jugular vein abnormalities and cerebral venous sinus thrombosis. Chin Med $\mathrm{J}$ (Engl) 2012;125:3671-3674.

6. Dentali F, Gianni M, Growter MA, et al. Natural history of cerebral vein thrombosis: a systematic review. Blood 2006;108:1129-1134.

7. Timóteo Â, Inácio N, Machado S, et al. Headache as the solo presentation of cerebral venous thrombosis: A prospective study. J Headache Pain 2012;13:487-490.

8. Kalita J, Chandra S, Misra UK. Signifiance of seizure in a cerebral venous sinus thrombosis. Seizure 2012;21:639-642.

9. Gosk-Bierska I, Wysokinski W, Brown RD, et al. Cerebral venous sinus thrombosis: Incidence of venous thrombosis recurrence and survival. Neurology 2006;67:814-819.

10. Fischer C, Goldstein J, Eldow J. Cerebral venous sinus thrombosis in the emergency department: retrospective analysis of 17 cases and review of the literature. J Emerg Med 2010;38:140-147.

11. Saposnik G, Barinagarrementeria F, Brown RD et al. Diagnosis and management of cerebral venous thrombosis: a statement for healthcare professionals from the American Heart Association/ American Stroke Association. Stroke 2011;42:1158-1172.

12. Alvis-Miranda HR, Castellar-Leones SM, Alcala-Cerra G, et al. Cerebral sinus venous thrombosis. J Neurosci Rural Pract 2013;4:427- 438.

13. Geisbüsch C, Lichy C, Richter D, et al. Clinical course of cerebral sinus venous thrombosis: data from a monocentric cohort study over 15 years. Nervenarzt 2014;85:211-220.

14. Uzar E1, Ekinci F, Acar A, et al. Cerebral venous sinus thrombosis: an analyses of 47 patients. Eur Rev Med Pharmacol Sci 2012;16:14991505.
15. deVeber G, Andrew M, Adams C, et al. Cerebral sinovenous thrombosis in children. N Engl J Med 2001;345:417-423.

16. Ameri A, Bousser MG. Cerebral venous thrombosis. Neurol Clin 1992;10:87-111.

17. de Freitas GR, Bogousslavsky J. Risk factors of cerebral vein and sinus thrombosis. Front Neurol Neurosci 2008;23:23-54.

18. Wysokinska EM, Wysokinski WE, Brown RD, et al. Thrombophilia differences in cerebral venous sinus and lower extremity deep venous thrombosis. Neurology 2008;70:627-633.

19. Brown A. Preventing venous thromboembolism in hospitalized patients with cancer: improving compliance with clinical practice guidelines, Am J Health Syst Pharm, 2012;69:469-481.

20. Dobbs TD, Barber ZE, Squier WL, et al. Cerebral venous sinus thrombosis complicating traumatic head injury. J Clin Neurosci 2012;19:1058-1059.

21. Mazzoleni R, Piette T, Lucas C, et al. Thrombose veineuse cérébrale au décours d'une meningo-radiculite herpétique. Quel est le mécanisme physiopathologique ? Rev Neurol (Paris) 2012;168:379-380.

22. Price K, Wilson L, Tsehaye M. A case of craniocervical abscess with sinus thrombosis in Lemierre's syndrome. Br J Neurosurg 2012;26:426-428.

23. Costa P, Del Zotto E, Giossi A, et al. Headache due to spontaneous intracranian hypotension and subsequent cerebral vein thrombosis. Headache 2012;52:1592-1596.

24. Fabricius J, Klotz JM, Hofmann E, et al. Cerebral venous thrombosis and subdural haematoma: complications of spontaneous intracranial hypotension. Fortschr Neurol Psychiatr 2012;80:599-601(Abstract).

25. Mao YT, Dong Q, Fu JH. Delayed subdural hematoma and cerebral venous thrombosis in a patient with spontaneous intracranial hypotension. Neurol Sci 201;32:981-983.

26. Eudo C. Petit A, Mondon K, et al. Cerebral venous sinus thrombosis in an individual with multiple myeloma treated with lenalidomide. J Am Geriatr Soc 2011;59:2371-2372.

27. Miki Y, Tomiyama M, Arai A, et al. Cerebral venous thrombosis with dural arteriovenous fistulas and antiphosholopid syndrome: a case report. Neurol Sci 2010;31:237-238.

28. Godfrey AL, Higgins JN, Beer PA, et al. In situ thrombolysis for cerebral venous thrombosis complicating anti-leukemic therapy. Leuk Res 2011;35:1127-1129.

29. Lizarazo-Barrela JC, Jacobelli S, Mellado P, et al. Extensive cerebral vein thrombosis as first manifestation of Behçet's disease. Report of one case. Rev Med Chile 2010;138:746-751 (Abstract).

30. Ahbeddou N, Benomar A, Rasmouni K, et al. Thrombose veineuse cérébrale et polyradiculonévrite aiguë révéelant un lupus érythémateux disséminé. Rev Neurol (Paris) 2010;166:458-463.

31. Casado-Menédes I, Uría DF, Jiménez L. Cerebral venous thrombosis as a complication following a diagnostic lumbar puncture. Rev Neurol 2011;52:252-253 (Abstract).

32. Skeik N, Stark MM, Tubman DE. Review Complicated cerebral venous sinus thrombosis with intracranial hemorrhage and mastoiditias. Vasc Endovascular Surg 2012;46:585-590.

33. Gao H, Yang BJ, Jin LP et al - Prdisposing factors, diagnosis, treatment and prognosis of cerebral venous thrombosis during and postpartum: a case-control study. Chin Med J (Engl) 2011;124:4198-4204.

34. Ruiz-Sandoval JL, Chiquete E, Bañuelos-Bacerra LJ, et al. Cerebral venous thrombosis in a Mexican multicenter registry of acute cerebrovascular disease: the RENAMEVASC study. J Stroke Cerebrovasc Dis 2012;21:395-400.

35. Coutinho JM , Ferro JM, Canhão P, et al. Cerebral venous and sinusal trombosis in womwn. Stroke 2009;40:2356-2361.

36. Sharma S, Sharma N, Yeoleker ME. Acute subarchnoid hemorrhage as initial presentation of dural sinus thrombosis. J Neurosci Rural Pract 2010;1:23- 25.

37. Canhão P, Ferro JM, Lindgren AG, et al. Causes and predictors of death in cerebral venous thrombosis. Stroke 2005;36:1720-1725

38. Lanska DJ, Kryscio RJ. Risk factors for peripartum and postpartum stroke and intracranial venous thrombosis. Stroke 2000;31:1274-1282.

39. Einhäupl K, Bousser MG, de Brujin SF, et al. EFNS guideline on the treatment of cerebral venous and sinus thrombosis. Eur J Neurol 2010;17: 1229-1235.

40. Daif A, Awada A, al-Rajeh S, et al. Cerebral venous thrombosis in adults. A study of 40 cases from Saudi Arabia. Stroke 1995;26:1193-1195.

41. Vijay RK. The cord sign. Radiology 2006;240:299-300.

42. Mahmoud M, Eliseblawy M. The role of multidetector CT venography in diagnosis of cerebral venous sinus thrombosis. Res $J$ Med Sci 2009;4:284-289.

43. Kim BS, Do HM, Marks MP. Diagnosis and management of cerebral venous and sinus Thrombosis. Semin Cerebrovasc Dis Stroke 2004;4:205-216. 
44. Chen HM, Chen CC, Tsai FY, et al. Cerebral sinovenous thrombosis. Neuroimaging diagnosis and clinical management. Interv Neuroradiol 2008;14:35-40.

45. El Damarawy EA, El-Nekiedy AE, Fathi AM, et al. Role magnetic resonance venography in evaluation of cerebral veins and sinuses occlusion. Alexandria J Med 2012;48:29-34.

46. Leach JL, Fortuna RB, Jones BV, et al. Imaging of cerebral venous thrombosis: Current techniques, spectrum of findings, and diagnostic pitfalls. Radiographics 2006;26:S19- S41.

47. Ayanzes RH, Bird CR, Keller PJ, et al. Cerebral MR venography: Normal anatomy and potential diagnostic pitfalls. Am J Neuroradiol 2000; 21:7478.

48. Yii IY, Mitchell PJ, Dowling RJ, et al. Imaging predictors of clinical deterioration in cerebral venous thrombosis. J Clin Neurosci 2012;19:15251529.

49. Coutinho JM, Ferro JM, Zuurbier SM. Thrombolysis or anticoagulation for cerebral venous thrombosis: rationale and design of the TO-ACT trial. Internat J Stroke 2013;8:135-140.

50. Coutinho JM, Stam J. How to treat cerebral venous and sinus thrombosis, J Thrombosis Haemostasis 2010;8:877-878.
51. Coutinho JM, Ferro JM, Canhão P, et al. Unfractionated or low - molecular weight heparin for the treatment of cerebral venous thrombosis. Stroke 2010;41:2575-2580.

52. Misra UK, Kalita J, Chandra S, et al. Low molecular weight heparin versus unfractionated heparin in cerebral venous sinus thrombosis: a randomized controlled trial. Eur J Neurol 2012; 19:1030-1036.

53. De Bruijn SFTM, Stam J. Randomized, placebo-controlled trial of anticoagulant treatment with low-molecular-weight heparin for cerebral sinus thrombosis. Stroke 1999;30:484-488.

54. Ferro JM, Canhão P, Stam J, et al. Prognosis of cerebral vein and dural sinus thrombosis. Stroke 2004;35:664-670.

55. Cumurciuc R, Crassard I, Sarov M, et al. Headache as the only neurological sign of cerebral venous thrombosis: a series of 17 cases. J Neurol Neurosurg Psychiatry 2005;76:1084-1087.

56. Libourel EJ, ten Kate MK, Brouwer JL, et al. Contribution of multiple thrombophilic and transient risk factors in the development of cerebral venous thrombosis. Thromb Res 2007;121:301-307.

57. Bhojo A, Wasay M, Saadah M, et al. Venous thrombosis. A descriptive multicenter study of patients in Pakistan and Middlde East. Stroke 2008;39:2707-2711. 